\title{
Disparate Effects of Insulin on Isolated Rabbit Afferent and Efferent Arterioles
}

\author{
Luis A. Juncos and Sadayoshi Ito \\ Hypertension and Vascular Research Division, Department of Internal Medicine and Heart and Vascular Institute, \\ Henry Ford Hospital, Detroit, Michigan 48202
}

\begin{abstract}
Despite evidence that insulin per se may be an important regulator of glomerular hemodynamics, little is known about its direct action on the glomerular afferent arterioles (Af-Art) and efferent arterioles (Ef-Art), the crucial vascular segments that control glomerular hemodynamics. In the present study, we examined the direct effect of physiological concentrations of insulin on isolated microperfused rabbit Af- and Ef-Arts. After cannulation, vessels were equilibrated in insulin-free medium for $\mathbf{3 0}$ min. To determine whether insulin causes vasodilation or constriction, increasing doses $(5,20$, and $200 \mu \mathrm{U} / \mathrm{ml})$ were added to the bath and lumen of arterioles that were either preconstricted to $50 \%$ of control diameter with norepinephrine or left nonpreconstricted. Insulin caused no vasoconstriction in either Af- or Ef-Arts, but it reversed norepinephrine-induced constriction in Ef-Arts but not Af-Arts (suggesting a vasodilator action selective to the Ef-Art ): at $200 \mu \mathrm{U} / \mathrm{ml}$, insulin increased Ef-Art luminal diameter by $75.8 \pm 7.0 \%$ from the preconstricted level ( $n=6 ; P<0.008)$. The vasorelaxant effect of insulin on Ef-Arts was not affected by blockade of either endotheliumderived relaxing factor/nitric oxide or prostaglandin synthesis. Despite the lack of effect of insulin on Af-Art when added after the equilibration period, when Af-Arts were equilibrated in the presence of either 20 or $200 \mu \mathrm{U} / \mathrm{ml}$ insulin, their basal diameter was significantly reduced $(11.7 \pm 0.9 \mu \mathrm{m} ; P<0.025, n=6$, and 12.0 $\pm 0.9 \mu \mathrm{m} ; P<0.025, n=7$, respectively) compared with nontreated Af-Arts $(16.2 \pm 1.3 \mu \mathrm{m} ; n=7)$. In conclusion, this study demonstrates that at physiological concentrations, insulin dilates NE-constricted Ef-Arts, while insulin pretreatment enhances Af-Art tone. The disparate actions of insulin on the Af- vs the Ef-Art may contribute to its beneficial effect on glomerular hypertension. (J. Clin. Invest. 1993. 92:19811985.) Key words: renal circulation • microvasculature • angiotensin II • diabetes • glomerular hemodynamics
\end{abstract}

\section{Introduction}

The early stages of both clinical and experimental diabetes mellitus (1-9) are characterized by an increase in the GFR. This hyperfiltration is mainly caused by an elevated glomerular plasma flow rate and increased glomerular capillary pressure (even in the presence of normal systemic blood pressure) (3,

Address correspondence to Sadayoshi Ito, M.D., Ph.D., Hypertension and Vascular Research Division, Henry Ford Hospital, 2799 W. Grand Boulevard, Detroit, MI 48202.

Received for publication 1 March 1993 and in revised form 19 May 1993

J. Clin. Invest.

(c) The American Society for Clinical Investigation, Inc.

$0021-9738 / 93 / 10 / 1981 / 05 \quad \$ 2.00$

Volume 92, October 1993, 1981-1985
6-9). Glomerular hypertension may be important in initiation and progression of diabetic nephropathy (10); consequently, several studies have attempted to discern the factors leading to glomerular hypertension in early diabetes. One factor that has been incriminated is insulin deficiency per se. Indeed, when insulin was infused into diabetic rats whose plasma glucose concentration was clamped at hyperglycemic levels, it lowered glomerular capillary pressure without affecting systemic blood pressure (7). In addition, the glomeruli are known to possess abundant insulin receptors (11-14) that could mediate actions besides glucose transport as in other vascular beds (15-21). Taken together, these findings suggest that insulin per se could be an important regulator of glomerular capillary pressure. Yet little is known about the direct effects of insulin on the primary regulators of glomerular capillary pressure, namely the glomerular afferent and efferent arterioles (Af- and Ef-Art). ${ }^{1}$

The purpose of the present study was to determine the direct effects of insulin on Af- and Ef-Arts in the absence of confounding influences such as tubuloglomerular feedback, neurohormonal influences, and changes in glucose concentrations. For this, we microdissected and perfused rabbit Af- and Ef-Arts in vitro and studied whether insulin per se causes vasodilation or constriction. Since we found that insulin dilated NE-constricted Ef-Arts, we next studied the role of PGs and endothelium-derived relaxing factor/nitric oxide (EDRF/ NO) in insulin-induced dilation of Ef-Arts. Finally, we studied whether insulin modulates the responses of Af-Arts to vasoconstrictors such as angiotensin II (Ang II) and NE.

\section{Methods}

\section{Isolation and microperfusion of Af- and Ef-Arts}

We isolated and microperfused Af- and Ef-Arts using methods similar to those described previously (22-25). Briefly, kidneys of young male New Zealand white rabbits (1.4-2.2 kg) were removed, sliced along the corticomedullary axis, and the slices were placed in ice-cold minimum essential medium (Gibco Laboratories, Grand Island, NY) containing 5\% BSA (Sigma Chemical Co., St. Louis, MO). From these slices, a single superficial Af-Art with its intact glomerulus was microdissected under an Olympus SZH stereomicroscope. Using a micropipette, the arteriole was transferred to a temperature-regulated chamber mounted on the stage of an Olympus IMT-2 or Nikon Diaphot inverted microscope with Hoffman modulation. The Af-Art was then cannulated with an array of glass pipettes. Intraluminal pressure was measured by the Landis technique, using a fine pipette introduced into the arteriole through the perfusion pipette, and was maintained at $60 \mathrm{mmHg}$ throughout the experiment.

Ef-Arts were perfused in an orthograde direction (24). For this, an Af-Art was microdissected together with the glomerulus and Ef-Art. The Af-Art was cut short $(\sim 50 \mu \mathrm{m})$ and cannulated as described

1. Abbreviations used in this paper: Af-Art, afferent arterioles; Ang II, angiotensin II; EDRF/NO, endothelium-derived relaxing factor/nitric oxide; Ef-Art, efferent arterioles; L-NAME, $\mathrm{N}^{\mathrm{w}}$-nitro-L-arginine. 
above, except that the perfusion pipette was advanced to the end of the Af-Art. The tip of the pressure pipette was placed just beyond the distal end of the Af-Art, and intraluminal pressure at this point was maintained at $40 \mathrm{mmHg}$ throughout the experiment to eliminate the hemodynamic influences of the Af-Art.

The arterioles were perfused with oxygenated medium 199 containing 5\% BSA. The bath was identical to the arteriolar perfusate (except that it contained $0.1 \%$ BSA) and was exchanged continuously. Microdissection and cannulation of the arteriole were completed within 90 min at $8^{\circ} \mathrm{C}$, after which the bath was gradually warmed to $37^{\circ} \mathrm{C}$ for the rest of the experiment. Once the temperature was stable, a 45-min equilibration period was allowed before taking any measurements. Images of the arteriole were displayed at magnifications $\leq 1,980$ and recorded with a Sony video system consisting of a camera (DXC-755), monitor (MA PVM-1942Q), and video recorder (EDV-9500). The diameter was measured with an image analysis system (Fryer, Carpentersville, IL).

\section{Experimental protocols}

Response of Af- and Ef-Arts to insulin. After the 45-min equilibration period, increasing doses of regular porcine insulin $(5-200 \mu \mathrm{U} / \mathrm{ml}$; Lilly Corp., Indianapolis, IN) were added to both bath and lumen. Luminal diameter was measured immediately before adding insulin and observed for $30 \mathrm{~min}$ at each dose.

Response of preconstricted arterioles to insulin. To determine whether insulin dilates Af- or Ef-Arts, arterioles were preconstricted to approximately $50 \%$ of basal diameter by adding NE (Sigma Chemical Co.) to the bath, after which increasing doses of insulin ( $5-200 \mu \mathrm{U} / \mathrm{ml}$ ) were added to both bath and lumen for $15 \mathrm{~min}$ at each concentration.

Effect of cyclooxygenase blockade or EDRF/NO synthesis inhibition on insulin-induced dilation of Ef-Arts. Since we found that insulin reversed NE-induced constriction of Ef- but not Af-Art (see Results), we next attempted to determine the mechanism by which insulin relaxes the Ef-Art. To test whether insulin-induced dilation of Ef-Arts is mediated by PGs, the cyclooxygenase inhibitor indomethacin (Sigma Chemical Co.) was added to the bath and arteriolar perfusate at a final concentration of $5 \times 10^{-5} \mathrm{M}$ from the equilibration period to the end of the experiment. After the equilibration period, Ef-Arts were preconstricted with NE as before, after which increasing concentrations of insulin were added to the bath and lumen for $15 \mathrm{~min}$ at each concentration. We have confirmed that this dose of indomethacin blocks the effect of arachidonic acid $\left(10^{-4} \mathrm{M}\right)$ in rabbit Af-Arts (26).

To determine whether EDRF/NO plays a role in insulin-induced dilation of Ef-Arts, we added the EDRF/NO synthesis inhibitor $N^{w}$-nitro-L-arginine methyl ester ( L-NAME, $10^{-4} \mathrm{M}$ ) (Sigma Chemical Co.) to the arteriolar perfusate after the equilibration period. The arteriole was then preconstricted with NE, after which increasing concentrations of insulin were added to the bath and lumen as above. We have previously reported that this concentration of L-NAME abolishes acetylcholine-induced vasodilation in this preparation $(23,24)$.

Effect of insulin pretreatment on Ang II- and NE-induced constriction in Af-Arts. We tested the possibility that even though insulin caused no constriction or dilation of Af-Arts, it could modulate vascular response to Ang II or NE. For this, dose-response curves for Ang II and NE were obtained in untreated control Af-Arts and in Af-Arts pretreated with either 20 or $200 \mu \mathrm{U} / \mathrm{ml}$ insulin. In these studies, insulin was added to both the bath and arteriolar perfusate and maintained throughout the experiment, including the cannulation procedure and equilibration period (in contrast to the first two protocols, where insulin was added after the equilibration period). Increasing concentrations of either Ang II (Sigma Chemical Co.) or NE were then added to the bath.

\section{Data analysis}

Values were expressed as mean \pm SEM. Paired $t$ tests were used to examine whether the diameter at a given concentration differed from the control value. To test whether groups differed with respect to the rate of change across the various periods, we used univariate repeated mea- sures ANOVA with the Greenhouse-Geisser sphericity correction. For this analysis, each experimental period was represented in terms of its percent change from the control period. Student's two-sample $t$ test was used to examine whether the baseline diameter differed between groups. When more than one comparison was made, Bonferroni's multiple comparison adjustment was used to reduce the significance level.

\section{Results}

Response of Af- and Ef-Arts to insulin. Basal luminal diameter of the Af-Arts was $14.8 \pm 0.80 \mu \mathrm{m}(n=5)$, while that of the Ef-Arts was $11.4 \pm 1.25 \mu \mathrm{m}(n=5)$. Addition of 5, 20, or 200 $\mu \mathrm{U} / \mathrm{ml}$ insulin had no effect on luminal diameter of Af-Arts $(14.5 \pm 0.85,14.9 \pm 0.82$, and $14.5 \pm 0.78 \mu \mathrm{m}$, respectively) or Ef-Arts $(11.7 \pm 1.10,12.2 \pm 1.12$, and $12.0 \pm 1.13 \mu \mathrm{m}$, respectively).

Response of preconstricted Af- and Ef-Arts to insulin. Fig. 1 shows an example of response induced by insulin in Ef-Arts. The top and middle panels show an Ef-Art in its basal state (Control) and after it has been preconstricted with NE, respectively. The lower panel shows the same arteriole after the addition of $200 \mu \mathrm{U} / \mathrm{ml}$ insulin for $15 \mathrm{~min}$. As clearly seen, insulin reversed NE-induced constriction in Ef-Art. Fig. 2 summarizes the responses of NE-preconstricted Af- and Ef-Arts to insulin. When insulin was added to Ef-Arts that were preconstricted

\section{Control}
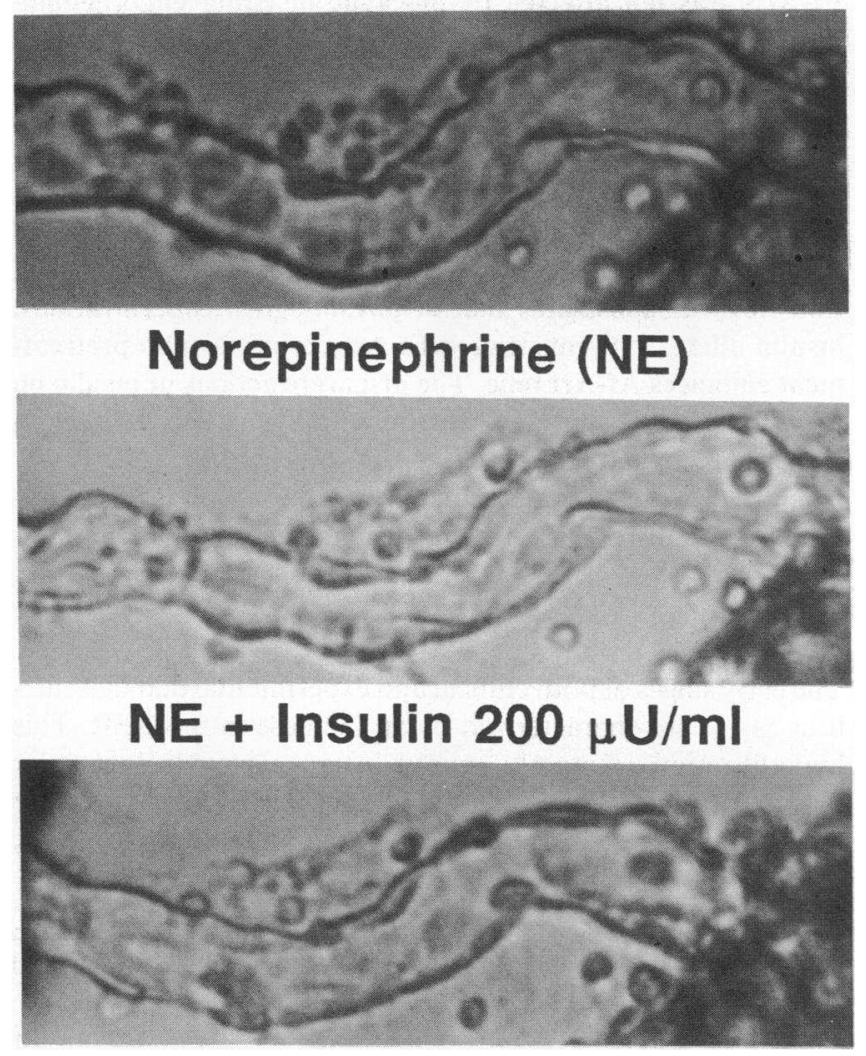

Figure 1. Example of arteriolar response to $200 \mu \mathrm{U} / \mathrm{ml}$ insulin in an Ef-Art preconstricted with norepinephrine. The top panel shows an Ef-Art in its basal state (Control). The middle panel shows the same arteriole preconstricted with NE, while the lower panel shows the arteriole after addition of $200 \mu \mathrm{U} / \mathrm{ml}$ insulin. Note the insulin-induced vasodilation. 


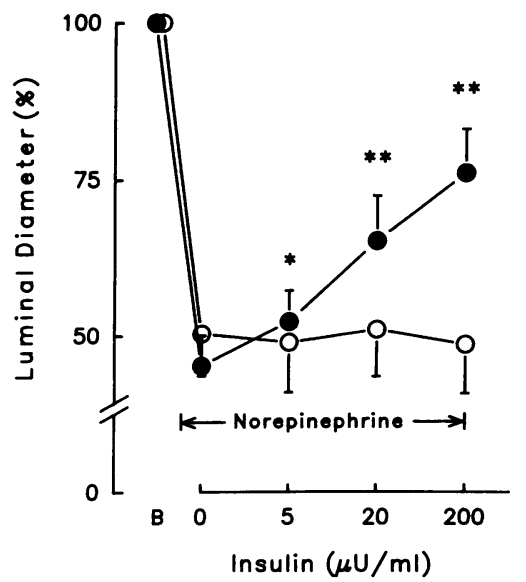

Figure 2. Effect of insulin on the luminal diameter of Af- $(0)(n$ $=5)$ and Ef-Arts $(\bullet)(n$ $=6)$ preconstricted with norepinephrine. ${ }^{*} P<0.017$ vs control diameter (NE without insulin). ${ }^{* *} P<0.008$ vs control diameter. with NE to $45.2 \pm 4.8 \%$ of basal diameter (from $11.5 \pm 0.85$ to $5.3 \pm 0.92 \mu \mathrm{m} ; n=6)$, luminal diameter increased in a dose-dependent manner to $6.1 \pm 0.93 \mu \mathrm{m}$ or $52.2 \pm 5.0 \%(P<0.017)$, $7.7 \pm 1.50 \mu \mathrm{m}$ or $65.1 \pm 7.2 \%(P<0.008)$, and $8.9 \pm 1.54 \mu \mathrm{m}$ or $75.8 \pm 7.0 \%(P<0.008)$ at 5,20 , and $200 \mu \mathrm{U} / \mathrm{ml}$ insulin, respectively. In marked contrast, insulin had no effect on Af-Arts; NE decreased luminal diameter from $16.8 \pm 1.13$ to $8.5 \pm 1.18 \mu \mathrm{m}$ (or $50.3 \pm 6.7 \%$ of basal, $n=5$ ), which remained unchanged after addition of 5,20 , and $200 \mu \mathrm{U} / \mathrm{ml}$ insulin $(8.3 \pm 1.56 \mu \mathrm{m}$ or $48.9 \pm 8.0 \%, 8.7 \pm 1.51 \mu \mathrm{m}$ or $50.9 \pm 7.5 \%$, and $8.3 \pm 1.60 \mu \mathrm{m}$ or $48.5 \pm 7.8 \%$, respectively).

Effect of cyclooxygenase blockade or EDRF/NO synthesis inhibition on insulin-induced dilation of Ef-Arts. The effect of inhibiting cyclooxygenase or EDRF/NO synthesis on insulininduced dilation of NE-preconstricted Ef-Arts is shown in Fig. 3. Basal diameter of indomethacin-pretreated Ef-Arts was $13.8 \pm 0.61 \mu \mathrm{m}(n=4)$. When insulin was added to Ef-Arts preconstricted to $7.9 \pm 0.25 \mu \mathrm{m}(57.4 \pm 1.3 \%$ of basal diameter $)$, diameter increased in a manner similar to the nontreated EfArts, becoming $7.8 \pm 3.8 \mu \mathrm{m}$ or $56.5 \pm 0.78 \%, 9.3 \pm 0.35 \mu \mathrm{m}$ or $68.0 \pm 1.9 \%$, and $11.6 \pm 0.84 \mu \mathrm{m}$ or $83.7 \pm 2.4 \%$, at 5,20 , and 200 $\mu \mathrm{U} / \mathrm{ml}$ insulin, respectively.

Likewise, inhibition of EDRF/NO synthesis did not affect insulin-induced dilation of NE-preconstricted Ef-Arts. After L-NAME pretreatment, Ef-Arts were preconstricted with NE to $53.6 \pm 4.0 \%$ of basal diameter (from 11.8 $\pm 1.63 \mu \mathrm{m}$ to $6.22 \pm 0.76 \mu \mathrm{m} ; n=4)$. As with the nontreated controls, insulin increased luminal diameter to $6.9 \pm 0.76 \mu \mathrm{m}$ or $60.5 \pm 7.4 \%$,

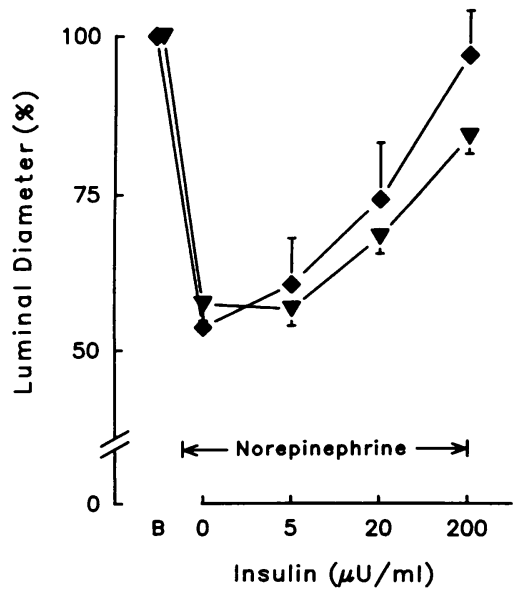

Figure 3. Effect of indomethacin $(\nabla)(n=4)$ and L-NAME pretreatment $(\bullet)(n=4)$ on insulin-induced dilation of Ef-Arts. Note that neither indomethacin nor L-NAME inhibited insulin-induced vasodilation.

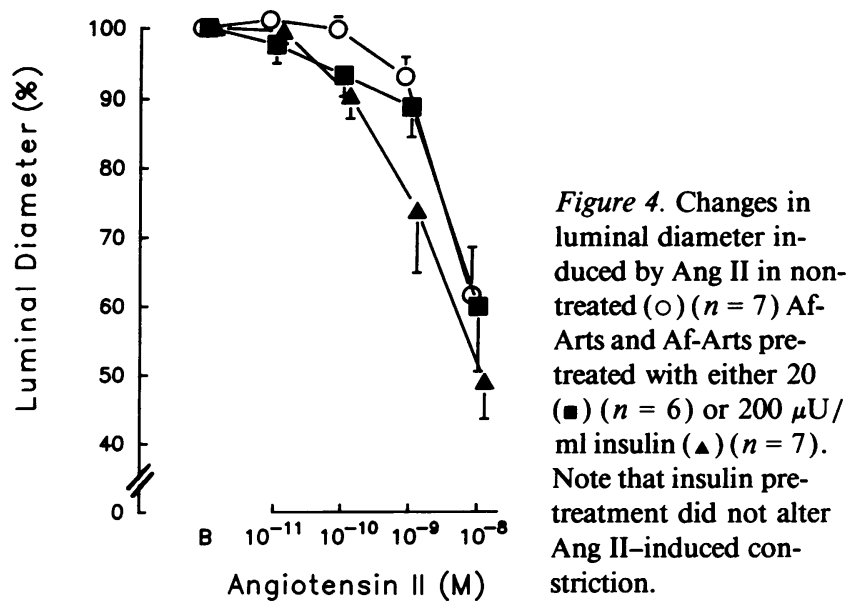

$8.4 \pm 0.70 \mu \mathrm{m}$ or $74.0 \pm 8.9 \%$, and $11.1 \pm 0.95 \mu \mathrm{m}$ or $96.5 \pm 7.0 \%$ at 5,20 , and $200 \mu \mathrm{U} / \mathrm{ml}$, respectively.

Effect of insulin pretreatment on Ang II- and NE-induced constriction in Af-Arts. Insulin pretreatment decreased basal diameter of Af-Arts (suggesting a vasoconstrictor action): basal diameter was $16.2 \pm 1.25 \mu \mathrm{m}(n=7)$ in nontreated Af-Arts, while it was $11.7 \pm 0.91(n=6, P<0.025)$ and $12.0 \pm 0.93(n$ $=7, P<0.025)$ in Af-Arts pretreated with 20 or $200 \mu \mathrm{U} / \mathrm{ml}$ insulin, respectively. However, insulin pretreatment did not significantly alter the Af-Art response to either Ang II (Fig. 4) or NE (Fig. 5), although Ang II-induced vasoconstriction tended to be augmented in Af-Arts pretreated with $200 \mu \mathrm{U} / \mathrm{ml}$ insulin, it was not statistically significant.

Since prolonged pretreatment, but not the shorter exposure to insulin caused Af-Arts to constrict, we tested whether prolonged pretreatment of Ef-Arts with insulin alters Ef-Art diameter. For this, we pretreated Ef-Arts with either $20 \mu \mathrm{U} / \mathrm{ml}$ insulin or control medium and measured basal diameters (we used only $20 \mu \mathrm{U} / \mathrm{ml}$ insulin in Ef-Arts because the decrease in basal diameter of Af-Arts was the same with $20 \mu \mathrm{U} / \mathrm{ml}$ or $200 \mu \mathrm{U} /$ $\mathrm{ml}$ ). Unlike in Af-Arts, insulin pretreatment did not affect basal diameter of Ef-Arts ( $13.4 \pm 0.12 \mu \mathrm{m}, n=5$ in nontreated Ef-Arts vs $13.2 \pm 0.37 \mu \mathrm{m}, n=5$ in insulin-pretreated Ef-Arts).

\section{Discussion}

The role of insulin as a glucoregulatory hormone, as well as its contribution to the regulation of cellular metabolism and ion

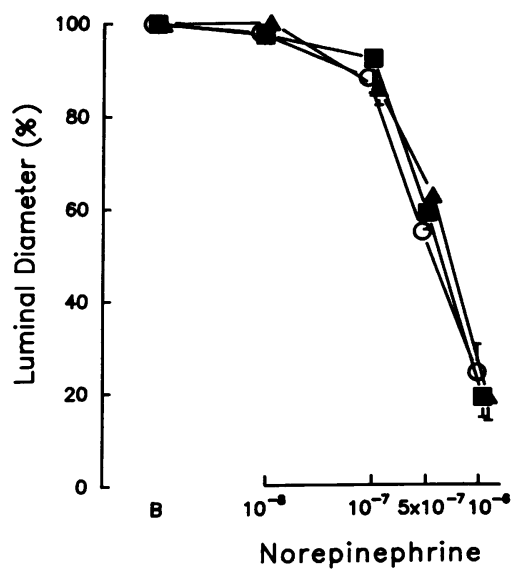

Figure 5. Changes in luminal diameter induced by Ang NE in nontreated $(0)(n=7)$ AfArts and Af-Arts pretreated with either 20 (घ) $(n=6)$ or $200 \mu \mathrm{U} /$ $\mathrm{ml}$ insulin $(\Delta)(n=7)$. Note that insulin did not significantly alter NE-induced constriction. 
transport, has been extensively studied. More recently, the role of insulin in other physiological functions, such as the regulation of blood pressure and regional blood flow, has received increased attention. Indeed, in view of the abnormalities of the glomerular circulation that occur in diabetes mellitus (1-10), it has been proposed that insulin may be an important regulator of the renal circulation $(7,25,26)$. However, it has been difficult to establish whether insulin-induced changes in renal hemodynamics are attributable to its direct vascular actions. With the development of techniques that permit direct visualization of the glomerular arterioles, it has become possible to study the direct vascular actions of insulin on these arterioles. In the present study, we provide the first direct measurements of the effect of insulin on the diameter of isolated glomerular arterioles.

The effect of insulin on renal hemodynamics in vivo in normal animals and humans is variable. While some studies showed no effect on either GFR or renal blood flow (27), others showed increases in GFR, renal blood flow, or both $(28-32)$. The present study shows that insulin markedly reverses NE-induced constriction in isolated microperfused EfArts but not Af-Arts; the effect was evident at concentrations as low as $5 \mu \mathrm{U} / \mathrm{ml}$. On the other hand, pretreatment of the glomerular arterioles with insulin reduced basal luminal diameter of Af-Arts by $25 \%$, but had no effect on the basal diameter of Ef-Arts, thus suggesting a paradoxical vasoconstrictive action on the Af-Art. Thus it appears that at physiological concentrations insulin per se has an effect on the glomerular arterioles, but this effect is strikingly different in Ef vs Af-Arts. However, our observations are not in complete agreement with the micropuncture study of Tucker et al (29). They found that in normal rats, infusion of insulin in quantities that require significant glucose infusion $(200-300 \mathrm{mg} / \mathrm{ml}$ per $\mathrm{h}$ ) resulted in renal vasodilation that was primarily caused by decreased Af-Art resistance. (Although Ef-Art resistance also decreased, it was not statistically significant). While the reason for these discrepancies is not clear, it may be related to differences in species, doses of insulin used, as well as the presence or absence of indirect actions of insulin. For example, insulin is known to stimulate sodium and/or chloride reabsorption in the proximal tubule $(33,34)$ and loop of Henle $(35)$. This would reduce the sodium chloride concentration at the macula densa, resulting in predominant Af-Art vasodilation through the mechanism called tubuloglomerular feedback (tubular fluid was collected at the distal tubule in Tucker's study). Furthermore, insulin may decrease renin release and Ang II levels within the kidney and also interact with vasoactive substances, which may have influenced the results. Indeed, Tucker et al. observed that an inhibitor of thromboxane $\mathrm{A}_{2}$ synthesis abolished insulininduced vasodilation.

To study the effect of insulin on renal function in the absence of systemic hemodynamic and hormonal influences, Cohen et al. (28) used isolated perfused kidneys from normal rats. When insulin was added to the perfusate beginning at the time of isolation, basal GFR and renal blood flow were significantly higher compared to kidneys perfused with insulin-free solution. These effects were observed with physiological concentrations ( $10-100 \mu \mathrm{U} / \mathrm{ml})$ and suggest that insulin predominantly dilates the Af-Art. However, when kidneys were first perfused with insulin-free solution and preconstricted with Ang II, addition of insulin (albeit at higher concentrations: $1,000 \mu \mathrm{U} / \mathrm{ml}$ ) caused renal vasodilation without altering GFR, thereby sug- gesting a predominant dilator effect on the Ef-Art, which is consistent with the present study. Their observations suggest that insulin altered Af-Art tone when it was given at the time of isolation, but not when it was added later. This finding is particularly interesting in view that we also found that Af-Art tone was affected by insulin pretreatment but not by adding insulin after the equilibration period. However, in contrast to Cohen's study, we found that insulin pretreatment constricted the AfArt. This effect was specific to Af-Arts, since insulin pretreatment did not affect basal diameter of Ef-Arts.

It is not clear why insulin reduced Af-Art diameter only when it was given from the onset of perfusion. It may be that a longer exposure to insulin might be needed to induce vasoconstriction, since Af-Arts were exposed to insulin for $\sim 100 \mathrm{~min}$ in the pretreated group but only $30 \mathrm{~min}$ when insulin was added after the equilibration period. Indeed, in coronary arteries insulin-induced contractile actions became apparent only after incubation for $120 \mathrm{~min}$ (18). We have not tested this possibility because of the time restraints imposed on our preparation. It is also possible that an endogenous cofactor that was depleted during the equilibration period may be needed for insulin to cause effects that lead to increased basal tone: thus adding insulin after the equilibration period would no longer cause vasoconstriction. Such interrelationships have been reported previously; for example, insulin-induced antinatriuresis in the rat is only manifested in the presence of vasopressin (36).

The mechanism by which insulin dilated Ef-Arts in our study is unclear. We found that blocking either EDRF/NO or PG synthesis (using L-NAME or indomethacin, respectively) failed to alter insulin-induced dilation of Ef-Arts. Thus our results suggest that neither EDRF/NO nor PGs are involved in insulin-induced dilation of Ef-Arts. In contrast, indomethacin reportedly inhibits insulin-induced renal vasodilation in the isolated perfused kidney (29). The reason for the discrepancy is not clear, but may be related to species and/or preparation differences (particularly the presence or absence of structures such as collecting tubules and medullary interstitial cells, which are major sites of $\mathrm{PG}$ production ). Other possible mechanisms by which insulin may induce dilation of Ef-Arts include (a) reduction of calcium influx and/or increase in calcium efflux in the Ef-Art vascular smooth muscle cells (VSMC) as reported in rat aortic VSMCs $(20,21)$; and $(b)$ hyperpolarization of VSMCs that results from insulin's stimulatory effects on $\mathrm{Na} / \mathrm{K}$ ATPase (37-40). Alternatively, since the Ef-Arts were perfused in an orthograde fashion through the glomerulus, it is possible that insulin acted upon glomerular cells, causing them to release a diffusible substance (other than EDRF/NO or PGs), which in turn dilated the Ef-Art.

This study supports the hypothesis that insulin per se can regulate glomerular hemodynamics. Thus, the increased glomerular plasma flow and capillary pressure that characterize the early stages of diabetes mellitus $(3,6-9)$ may be at least partially caused by insulinopenia (or insulin resistance). Indeed, Scholey and Meyer (7) reported that infusion of insulin into diabetic rats lowered glomerular capillary pressure, whether plasma glucose concentration was clamped at the hyperglycemic level or was allowed to fall to the normal range. While this suggests that insulin may have directly affected glomerular hemodynamics, involvement of hormonal changes was not ruled out. The insulin-induced decrease in glomerular capillary pressure was caused by an increased ratio of Af- vs Ef-Art resistance (predominantly because of a fall in Ef-Art 
resistance). This pattern of glomerular vascular reactivity to insulin is quite consistent with that found in the present study, namely Af-Art constriction with Ef-Art dilation. Thus these data support the concept that insulin decreases glomerular capillary pressure by acting directly upon the glomerular arterioles. However, comparison of our finding with reported insulin's effect in diabetic animals in vivo requires caution, since vascular response to insulin may differ in normal vs diabetic animals or even in diabetes of different duration $(29,41)$.

In summary, we have shown that physiological concentrations of insulin have direct actions on glomerular arterioles that are markedly different in Af- vs Ef-Arts. Insulin caused dilation of Ef-Arts (but not Af-Arts) that was independent of EDRF/ NO and PGs. In addition, insulin pretreatment decreased basal diameter but had no effect on Ang II- or NE-induced constriction in Af-Arts. These results suggest that insulin per se may be important in the control of glomerular hemodynamics. Its dilatory action on the Ef-Art, coupled with its constrictor action on the Af-Art, may partially account for its beneficial effect on glomerular hypertension.

\section{Acknowledgments}

This study was supported by National Institutes of Health grants HL46518 and HL-28982. We gratefully acknowledge the technical assistance of Janki Amin, M.D.

\section{References}

1. Christiansen, J. S., J. Gammelgaard, M. Frandsen, and H.H. Parving 1981. Increased kidney size, glomerular filtration rate and renal plasma flow in short-term insulin-dependent diabetics. Diabetologia. 20:451-456.

2. Hostetter, T. H. 1991. Diabetic nephropathy. In The Kidney. 4th ed. B. M. Brenner and F. C. Rector, editors. W. B. Saunders Co., Philadelphia. pp. 16951726.

3. Hostetter, T. H., J. L. Troy, and B. M. Brenner. 1981. Glomerular hemodynamics in experimental diabetes mellitus. Kidney Int. 19:410-415.

4. Jensen, P. K., J. S. Christiansen, K. Steven, and H.-H. Parving. 1981. Renal function in streptozotocin-diabetic rats. Diabetologia. 21:409-414.

5. Michels, L. D., M. Davidman, and W. F. Keane. 1981. Determinants of glomerular filtration and plasma flow in experimental diabetic rats. J. Lab. Clin. Med. 98:869-885.

6. Zatz, R., R. Dunn, T. W. Meyer, S. Anderson, H. G. Rennke, and B. M. Brenner. 1986. Prevention of diabetic glomerulopathy by pharmacological amelioration of glomerular capillary hypertension. J. Clin. Invest. 77:1925-1930.

7. Scholey, J. S., and T. W. Meyer. 1989. Control of glomerular hypertension by insulin administration in diabetic rats. J. Clin. Invest. 83:1384-1389.

8. Jensen, P. K., K. Steven, H. Blaehr, J. S. Christiansen, and H.-H. Parving. 1986. Effects of indomethacin on glomerular hemodynamics in experimental diabetes. Kidney Int. 29:490-495.

9. Bank, N., R. Klose, H. S. Aynedjian, D. Nguyen, and L. B. Sablay. 1987. Evidence against increased pressure initiating diabetic nephropathy. Kidney Int. 31:898-905.

10. Hostetter, T. H., H. G. Rennke, and B. M. Brenner. 1982. The case for intrarenal hypertension in the initiation and progression of diabetic and other glomerulopathies. Am. J. Med. 72:375-380.

11. Kurokawa, K., F. J. Silverblatt, K. L. Klein, M. S. Wang, and R. L. Lerner. 1979. Binding of ${ }^{125} \mathrm{I}$-insulin to the isolated glomeruli of rat kidney. J. Clin. Invest. 64:1357-1364.

12. Abrass, C. K., G. J. Raugi, L. S. Gabourel, and D. H. Lovett. 1988. Insulin and insulin-like growth factor I binding to cultured rat glomerular mesangial cells. Endocrinology. 123:2432-2439.

13. Bulton, D., S. Vadrot, S. Roseau, and F. Morel. 1988. Insulin receptors along the rat nephron: ${ }^{125} \mathrm{I}$-insulin binding in microdissected glomeruli and tubules. Pfluegers Arch. 412:604-612.

14. Elliot, S. J., F. G. Conti, L. J. Striker, and G. E. Striker. 1990. Mouse glomerular endothelial cells have an insulin receptor. Horm. Metab. Res. 22:557560.
15. Cavaliere, T. A., and D. G. Taylor. 1981. The effects of insulin on vasoconstrictor responses in pithed rats. J. Clin. Pharmacol. 21:275-279.

16. Creager, M. A., C. S. Liang, and J. D. Coffman. 1985. Beta-adrenergic-mediated vasodilator response to insulin in the human forearm. J. Pharmacol. Exp. Ther. 235:709-714.

17. Yagi, S., S. Takata, H. Kiyokawa, M. Yamamoto, Y. Noto, T. Ikeda, and N. Hattori. 1988. Effects of insulin on vasoconstrictive responses to norepinephrine and angiotensin II in rabbit femoral artery and vein. Diabetes. 37:10641067.

18. Miwa, A. Y., H. Ito, and T. Sugimoto. 1990. Effects of insulin on vasoconstriction induced by thromboxane $\mathrm{A}^{2}$ in porcine coronary artery. Circulation. 81:1654-1659.

19. Townsend, R. R., R. Yamamoto, M. Nickols, D. J. Dipette, and G. A. Nickols. 1992. Insulin enhances pressor responses to norepinephrine in rat mesenteric vasculature. Hypertension (Dallas). 19(Suppl. II):II-105-II-110.

20. Standley, P. R., F. Zhang, J. L. Ram, M. B. Zemel, and J. R. Sowers. 1991. Insulin attenuates vasopressin-induced calcium transients and a voltage-dependent calcium response in rat vascular smooth muscle cells. J. Clin. Invest. 88:1230-1236.

21. Kim, Y. C., and M. B. Zemel. 1992. Insulin potentiates vascular smooth muscle cell (VSMC) intracellular $\mathrm{Ca}^{2+}\left(\left[\mathrm{Ca}^{2+}\right]\right)$ responses to angiotensin II but accelerates recovery from $\left[\mathrm{Ca}^{2+}\right]$ loads. FASEB (Fed. Am. Soc. Exp. Biol.) J. 6:A945. (Abstr.)

22. Ito, S., and O. A. Carretero. 1990. An in vitro approach to the study of macula densa-mediated glomerular hemodynamics. Kidney Int. 38:1206-1210.

23. Ito, S., C. S. Johnson, and O. A. Carretero. 1991. Modulation of angiotensin II-induced vasoconstriction by endothelium-derived relaxing factor in the isolated microperfused rabbit afferent arteriole. J. Clin. Invest. 87:1656-1663.

24. Ito, S., S. Arima, Y. Ren, L. A. Juncos, and O. A. Carretero. 1993. Endothelium-derived relaxing factor/nitric oxide modulates angiotensin II action in the isolated microperfused rabbit afferent but not efferent arteriole. J. Clin. Invest. 91:2012-2019.

25. Osgood, R. W., M. Patton, M. J. Hanley, M. Venkatachalam, H. J. Reineck, and J. H. Stein. 1983. In vitro perfusion of the isolated dog glomerulus. Am. J. Physiol. 244:F349-F354.

26. Itoh, S., and O. A. Carretero. 1985. Role of the macula densa in renin release. Hypertension (Dallas). 7(Suppl. I):I49-I54.

27. Bank, N., M. A. Lahorra, and H. S. Aynedjian. 1987. Acute effect of calcium and insulin on hyperfiltration of early diabetes. Am. J. Physiol. 252:E13E20.

28. Cohen, A. J., D. M. McCarthy, and J. S. Stoff. 1989. Direct hemodynamic effect of insulin in the isolated perfused kidney. Am. J. Physiol. 257:F580-F585

29. Tucker, B. J., C. M. Anderson, R. S. Thies, R. C. Collins, and R. C. Blantz 1992. Glomerular hemodynamic alterations during acute hyperinsulinemia in normal and diabetic rats. Kidney Int. 42:1160-1168.

30. Stenvinkel, P., J. Bolinder, and A. Alvestrand. 1992. Effects of insulin on renal hemodynamics and tubular $\mathrm{Na}$ handling in healthy man. J. Am. Soc. Nephrol. 3:569. (Abstr.)

31. Hall, J. E., M. W. Brands, S. D. Kivlighn, H. L. Mizelle, D. A. Hildebrandt, and C. A. Gaillard. 1990. Chronic hyperinsulinemia and blood pressure Interaction with catecholamines? Hypertension (Dallas). 15:519-527.

32. Hall, J. E., M. W. Brands, H. L. Mizelle, C. A. Gaillard, and D. A. Hildenbrandt. 1991. Chronic intrarenal hyperinsulinemia does not cause hypertension. Am. J. Physiol. 260:F663-F669.

33. DeFronzo, R. A., C. R. Cooke, R. Andres, G. R. Falcona, and P. J. Davis. 1975. The effect of insulin on renal handling of sodium, potassium, calcium, and phosphate in man. J. Clin. Invest. 55:845-855.

34. DeFronzo, R. A., M. Goldberg, and Z. S. Agus. 1976. The effects of glucose and insulin on renal electrolyte transport. J. Clin. Invest. 58:83-90.

35. Kirchner, K. 1988. Insulin increases loop segment chloride reabsorption in euglycemic rats. Am. J. Physiol. 255:F1206-F1213.

36. Clark, R. V., A. K. Gupta, and K. A. Kirchner. 1991. Vasopressin is an essential cofactor for insulin antinatriuresis in the rat. J. Am. Soc. Nephrol. 2:720. (Abstr.)

37. Zierler, K. L., and E. M. Rogus. 1981. Effects of peptide hormones and adrenergic agents on membrane potentials of target cells. Fed. Proc. 40:121-124.

38. Moore, R. D., and J. L. Rabovsky. 1979. Mechanism of insulin action on resting membrane potential of frog skeletal muscle. Am. J. Physiol. 236:C249254.

39. Zierler, K. L. 1957. Increase in resting membrane potential of skeletal muscle produced by insulin. Science (Wash. DC). 126:1067-1068.

40. Zierler, K. L. 1959. Effect of insulin on membrane potential and potassium content in rat muscle. Am. J. Physiol. 197:515-523.

41. Cohen, A. J., and D. M. McCarthy. 1988. Paradoxical effects of insulin in diabetic and non-diabetic perfused kidneys. Kidney Int. 33:410. (Abstr.) 